EGU2020-10407

EGU General Assembly 2020

(c) Author(s) 2020. This work is distributed under

the Creative Commons Attribution 4.0 License.

\title{
Rainfall estimate using Commercial Microwave Links (CML): first outcomes of the MOPRAM project
}

\author{
Roberto Nebuloni ${ }^{1}$, Michele D'Amico ${ }^{2}$, Greta Cazzaniga ${ }^{3}$, Carlo De Michele ${ }^{3}$, and Cristina Deidda ${ }^{3}$ \\ ${ }^{1}$ IEIIT, Consiglio Nazionale Delle Ricerche, Italy (roberto.nebuloni@ieiit.cnr.it) \\ ${ }^{2}$ DEIB, Politecnico di Milano, Italy (michele.damico@polimi.it) \\ ${ }^{3}$ DICA, Politecnico di Milano, Italy (carlo.demichele@polimi.it)
}

The measurement of space-time rainfall fields is of great importance for several purposes including weather forecast, water resource management, evaluation of hydrological risk and monitoring of hydrologic extremes. Conventional methods for rainfall measurement include rain gauges and weather radars, both types of sensor having their own advantages and limitations. A different and not fully tested methodology exploits the power loss (namely, the attenuation) experienced by microwave radio signals when travelling across rain along either terrestrial or ground-to-satellite links. Indeed, it is well known that microwave attenuation due to rain can be calculated from the rain rate along the propagation path. The inverse problem can be solved once the disturbances affecting the radio signal and not induced by rain have been identified and removed.

In this contribution, we present the first results of the experimental campaign carried out in the framework of MOPRAM (MOnitoring Precipitation through a Network of RAdio links at Microwaves). MOPRAM is a scientific project funded by Fondazione Cariplo, which aims at assessing the potential of Commercial Microwave Links (CML) for rainfall estimates and rainfall field retrieval in areas of hydrological interest located in Northern Italy. A network of CMLs, owned by a major mobile operator in Italy, has been exploited to estimate the average rain rate along each link. The available data are the minimum and maximum values of the transmitted and received signal power across each link (two-ways), measured during 15-min time slots.

We start by describing the procedure adopted for the estimation of the baseline, i.e. the received power level immediately before and after a precipitation event. Rain attenuation is subsequently calculated by subtracting the received power during the event from the baseline level. To this aim, every 15-min slot of each link is classified in advance as either wet or dry by taking advantage of the spatial correlation of rain. Path-averaged rainfall intensity can be retrieved from rain attenuation by formulas based on well-known electromagnetic models. Finally, CML-based rainfall is compared with the one obtained from co-located rain gauges and disdrometers.

Despite the CML setup is not optimized for rainfall measurements, preliminary results highlight a good correlation between the occurrence of wet periods detected by CMLs on one side and by rain gauges and disdrometers on the other, as well as a fair agreement between the corresponding time series of accumulated precipitation. 
